\title{
Editorial
}

\section{Cost Effective Use of Biological Therapy in Indian Scenario}

\section{J agdish RK*}

Consultant Rheumatologist, Kailash and Max Hospital, Noida and Ex. Senior Resident Rheumatology, AIIMS, Delhi, India

*Corresponding author: J agdish RK, Consultant Rheumatologist, Kailash and Max Hospital, Noida and Ex. Senior Resident Rheumatology, AIIMS, Delhi, India

Received: October 12, 2017; Accepted: October 30, 2017; Published: November 06, 2017

\section{Introduction}

The use of biological therapy in rheumatology and other practices is on rise in recent years, which attributable to many factors, firstly increased awareness of their use among doctors, among patients and in medical literature with time. Secondly availability of these agents at relatively less cost than earlier since the introduction of biosimilars. Currently there is no definitive consensus for the duration of therapy and about when to stop. Their cost effectiveness can be increased by proper and timely tapering particularly in low economic countries like India. There are some studies [1-4] available regarding tapering and withdrawing of biologicals in rheumatoid arthritis and other rheumatological illness.

\section{Study Methodology}

We done this retrospective study, done from March 2016 to July 2017, at single tertiary care centre in Noida, India, where 36 patients between the age of 15 to 85 years with different rheumatological diseases having poor response or dissatisfaction with first line medicines and who has received biological therapy were included in the study.

\section{Results}

Demographic and clinical parameters were evaluated as follows:

Demographic profile: Average age of study population was 50.86 years, $58.33 \%$ patients were female. Rheumatoid arthritis was most common primary diagnosis in $47.22 \%$ cases, followed by spondyloarthritis in $44.44 \%$ cases and $8.33 \%$ other cases. Average duration of diseases was 8.83 years, average duration of first line treatment before biological therapy was 5.75 years.
Biologicals used in the study population: Anti-TNF was the most common class of biologicals used in $77.77 \%$ of cases (etanercept in $47.22 \%$, adalimumab in $25 \%$, infliximab in $5.55 \%$ ). Rituximab was used in $19.44 \%$ cases and secukinumab in one case. Average duration of biological therapy was 15.92 months.

Biological tapering and possible stopping: Biological tapering was successfully done in $33.33 \%$ of cases (in $22.22 \%$ of cases dosing interval increased and $11.11 \%$ cases were able to stop biological). Biologicals stopped in $22.22 \%$ of cases (due to successful tapering in $11.11 \%$ cases, biological failure in $5.55 \%$ cases and side effects in $5.55 \%$ cases).

Response to treatment: Response to treatment was earliest and most sustained with rituximab. Biological failure occurs in 5.55\% $(n=2)$ of cases, one primary failure with adalimumab in rheumatoid arthritis, and one secondary failure with etanercept in psoriatic arthritis, which then treated with secukinumab.

Side effects: Minor side effects were noticed during rituximab infusion in two cases with throat irritation and chocking sensation while infusion, which got subsided with decreasing the infusion rate. Major side effect which leads to stopping the medicine were observed in two cases, one etanercept (non-healing cellulitis) and one adalimumab (non tubercular lymphadenopathy). Overall toxicity was low and manageable.

\section{Conclusion}

We can conclude that wiser use of biological agents can help to stop and taper the biologicals in cost constrained settings.

\section{References}

1. Allaart CF, Lems WF, Huizinga TW. The Best way of withdrawing biologic agents. Clin Exp Rheumatol. 2013; 31: S14-18.

2. Tanaka $Y$, Hirata S, Saleem B, Emery P. Discontinuation of biologics in patients with rheumatoid arthritis. Clin Exp Rheumatol. 2013; 31: S22-27.

3. Tanaka $Y$, Hirata $S$. Is it possible to withdraw biologics from therapy in rheumatoid arthritis? Clin Ther. 2013; 35: 2028-2035.

4. Tanaka $\mathrm{Y}$ et al, Adalimumab discontinuation in patients with early rheumatoid arthritis who were initially treated with methotrexate alone or in combination with adalimumab: 1 year outcomes of the HOPEFUL-2 study. RMD Open. 2016; 2: e000189. 